1 Dr. Ishrat Bhuiyan
MBBS, DDV, FCPS
Assistant Professor
Dept. of Dermatology \& Venereology
Shaheed Suhrawardi Medical
College and Hospital
20r. Md. Shahadat Hossain
MBBS, DDV, FCPS
Associate Professor
Dept. of Dermatology \& Venereology
Shaheed Suhrawardi Medical
College and Hospital
30r. Md. Moksedur Rahman
MBBS, DDV, MD
Associate Professor
Dept. of Dermatology \& Venereology
Rajshahi Medical College and Hospital
4 Dr. Yasmeen Joarder
MBBS, DDV
Associate Professor
Dept. of Dermatology \& Venereology
Northern International Medical
College, Dhaka

Correspondence

Dr. Ishrat Bhuiyan MBBS, DDV, FCPS

Assistant Professor

Dept. of Dermatology \& Venereology, Shaheed Suhrawardi Medical College and Hospital email: ishratskin@yahoo.com

\title{
Pityriasis amiantacea : A reaction pattern of the scalp in psoriatic patient
}

\author{
I Bhuiyan' ${ }^{1}$ M S Hossain², M M Rahman ${ }^{3}$, Y Joarder ${ }^{4}$
}

\section{Introduction}

Tinea amiantacea or pityriasis amiantacea (asbestos- like tinea) is a disease of scalp with heavy scales extend on to the hairs and separate and bind together their proximal portions. ${ }^{1}$ The process may be only slightly inflammatory with dry silvery, micaceous scales, overlying like the tiles on a roof ${ }^{1,2}$, adhere to the scalp or markedly inflammatory with admixture of crusts. ${ }^{1}$

According to the Alibert, inventor of the name of the condition, there is an early moist stage, followed by drier one. ${ }^{1}$ Removal of this scales reveals erythematous, edematous epidermis. ${ }^{1,2}$

The disease may be localized or diffuse. ${ }^{1,2,3}$ It is a chronic condition, but curable. ${ }^{1}$

This condition can occur at any age, specially during childhood, adolscents and young females. ${ }^{1,3}$

There is some amount of alopecia, non scarring in nature 2,3 but no atrophy and scarring. 1,2,3

The cause of pityriasis amiantacea is obscure. ${ }^{1}$ Many conditions are associated with pityriasis amiantacea including psoriasis, Seborrheic dermatitis, atopic dermatitis, tinea, pyoderma, pediculosis, lichen planus, Darier's disease. ${ }^{1,4}$

One Scandinavian study ${ }^{5}$, Psoriasis related in $15 \%$ of patients with Tinea amiantacea, but another study ${ }^{6}$ reveals psoriasis associated with $35 \%$ and eczematous conditions associated with $34 \%$ of cases.

Staphylococcus aureus, was isolated in many of the cases of pityriasis amiantacea. ${ }^{6,7}$

The importance of recognizing the underlying etiology may have a therapeutic implication. A limited number of reports of this entity have been published in the literature and some of them have discussed the etiology of this condition.

\section{Case Report}

In $21^{\text {th }}$ April, 2015, a 27 years old, unmarried, hotelboy, working at Maldives presented to the Department of Dermatology and Venereology, Shaheed Suhrawardi Medical College Hospital, Dhaka, Bangladesh with 3 months history of thick, slightly foul smelly, adherent scales that surround and bound down the tufts of hair involving the entire scalp. Patient also complaints of numerous well circumscribed,dry, silvery, thick scaly plaques of various size and shapes situated over the extensor aspects of lower extremities and back for last 9 months. This patient was also suffering from fine powdery scaly, slightly pruritic lesions on the scalp for last 6 months.

He stated that he was in Maldives for last 3 years. For this problem, he was treated with various antibiotics, antihistamins, anti-fungal, steroids, shampoos at Maldives but no improvement occured.

On examination, thick, slightly foul smelling silvery, micaceous, asbestos like adherent scaly plaques involving the whole scalp from frontal to occipital region were seen. Removal of the scales was not so easy. After removal of the scales the underlying epidermis was edematous, erythematous. The involved hairs are matted within adherent scales. Along with this there was no corresponding lymphadenopathy. Other systemic examination revealed no abnormality.

Skin biopsy was taken from the extremity lesion and showed hyperkeratosis, parakeratosis, munromicroabscesses in the stratum corneum, thining of the granular layer, thining of the supra papillary plate, dilatation and tortusity of the upper dermal blood vessels - features consistent with psoriasis. Another scalp biopsy revealed, diffuse hyperkeratosis and parakeratosis together with follicular keratosis, which surround each 
hair with a sheath of horn. Another scalp tissue sample was taken for $\mathrm{KOH}$ preparation, fungal culture showed no fungal positivity. Other hematological and biochemical parameters including Anti $\mathrm{HIV}_{1}$ and $\mathrm{HIV}_{2}$ were normal. Except slight neutrophilic leucocytosis, coagulase test positive for Staphylococcoi, so based on clinical examination \& investigation, a diagnosis of pityriasis amiantacea due to psoriasis was confirmed.

After curing the etiological factors, microscopic examination has shown normal hairs, with completely cornified epithelial cells in the scales.

Topical treatment was given with Tar shampoos and salicylic acid for scalp lesions; salicylic acid and steroid ointments combinations for body lesions. Orally tablet azithromycin 500mg daily for 2 weeks along with oral Methotrexate $10 \mathrm{mg}$ weekly were started. A good response was seen by the end of 1 month treatment. Frequency of tar shampoo, steroid ointment was gradually tapered and stopped after 2 months. The Methotrexate was continued in the same way for total 3 months.

No clinical relapse was found.

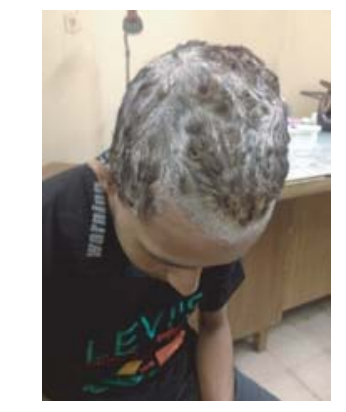

Thick, adherent silvery scales on entire scalp

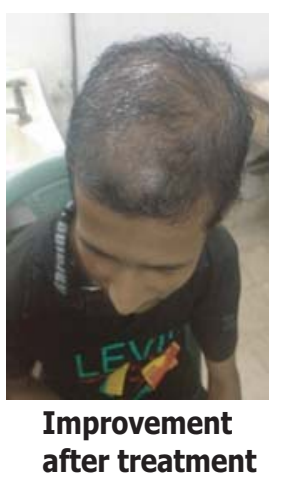

\section{Discussion}

To identify the etiology of pityriasis amiantacea puzzled the dermatologists and some studies have been made to reveal a definitive cause. However these attempts were limited in number conducted on a smaller number of patients or not inclusive of microbiological and histopathological examinations. The Ibrahim -Abdel- Hamid et,al ${ }^{6}$ conducted on a large number of patients with concomitant microbiological and histopathological examinations and indicate that pityriasis amiantacea represents a particular reaction pattern of the scalp to various inflammatory scalp diseases.

Fiften (15\%) patient in Ibrahim- Abdel-Hamid Studies showed psoriasis is associated with pityriasis amiantacea. The Knight $\mathrm{AG}^{7}$ Hansted and Lindskov ${ }^{8}$ studies found that pityriasis amiantacea could be the first clinical manifestation of psoriasis.

Hansted and Lindskov ${ }^{8}$ studies also discovered that predisposition to psoriasis in patients with pityriasis amiantacea was significantly higher than that of the general population. Therefore our study regarding presentation, etiology showed similarity with the mentioned studies.
An important considerations in diagnosis of pityraisis amiantacea is to ruleout the possibility of tinea capitis. Perhaps the most difficult task to findout the pathogenesis of pityriasis amiantacea is why the scalp reacts with approximately the same pattern to different diseases. Here the isolated Staphylococcus aureus in the lesion is either as a secondary infection as reported by Knight $\mathrm{AG}^{7}$ or a normal skin flora as observed by Mclinley et $\mathrm{al}^{9}$ could play an important role in the pathogenesis of pityriasis amiantacea. Staphylococcus aureus could produce an epidermal cell differentiation inhibitor ${ }^{10}$, which can be involved in inhibition of calcium induced terminal differentiation of human keratinocytes in culture leading to parakeratosis and hyperplasia of the epidermis. ${ }^{11}$ Further more, it has been observed that the total number of bacteria isolated from psoriatic plaques, a disease characterized by hyperkeratosis and parakeratosis was significantly higher than on the adjacent normal skin. ${ }^{12}$ This findings could have a therapeutic implication through the use of antibiotics to treat and or prevent recurrency of pityriasis amiantacea.

Although pityriasis amiantacea is commonly a localized condition but more than half of the patients of Ibrahim- Abdel Hamid study showed widespread disease.

Conclusion : Pityriasis amiantacea represents a particular reaction pattern of the scalp to various inflammatory scalp diseases. The most frequent skin diseases associated with pityriasis amiantacea are psoriasis. It is important to keep the diagnosis of tinea capitis in mind when evaluating pityriasis amiantacea patients. Staphylococcoi on the scalp could participate in the pathogenesis of pityriasis amiantacea

\section{References}

1. Becker SW, Muir KB. Tinea amiantacea. Arch DermatolSyphil 1929; 20: 45-53.

2. C. E. M .Griffihs\& J.N. W.N Barker. Psoriasis . In Rook's Text book of Dermatology.EDn 8th, vol-1, Edited by BurnsT, Breathnach S, Cox N, Griffiths C, Blackwell publishing. U.S.A, UK, Australia,2010:20.14-20.16

3. Chris D, Collins\& chad Hivnor, Seborrheic dermatitis. I Fitzpatrick's Dermatology in General Medicine. Edn8 ${ }^{\text {th }}$, vol-1. McGraw Hill, New York, Chicago, Sanfrancisco, Lisbon, London, Madrid, Mexico city, Milan, New Delhi, San Juan, Seol, Singapore, Sydney, Torento. 2012; 263-264

4. Daniel S. Ring, MD, David L. Kaplan, MD. PityraisisAmiantacea : A report of 10 cases.

5. Hansted B, Lindoskov R. Pityriasisamiantacea and Psoriasis : A follow up study. Dermatologica 1983; 166:314-315

6. Ibrahim A. Abdel - Hamid, MD, Salah ., Agha ,MD. Yospy M. Moustafa, MD and Aynan M. EL- Labban, MSC.pityriasisamiantacea: a clinlcal andetiopathological study of 85 patients. Int J Dermatol 2003; 42: 260-264

7. Knight AG. Pityriasisamiantacea: A Clinical and histopathological investigation. clinical Experience Dermatol 1977; 2: 137-142.a clinical and histopathologicalAmiantacea and Psoriasis : a follow p study, Dermatological 1983;166: 314-315

8. Hansted B, Lindoskov R. Pityriasisamiantacea and Psoriasis : A follow up study. Dermatologica 1983; 166:314-315

9. McGinley KJ,Leyden JJ, MarplesRR,Kligman AM. Quantitative microbiology of the scalp in non-dandruff,dandruff and seborrheic dermatitis. J Invest Dermatol 1975; 64:401-405

10. Sugai $M$, Enomoto $T$, Hashimoto $K$,et al. Epidermal Cell differentiation (EDIN): Purification and chacterization from staphylococcus aureus. BiochemBiophys RCs common 1990; 173-:92-98

11. Sugai M, Hashimoto K, Kikochi A,et al. Epidermal cell differentiation inhibitor ADP- ribosylates small GTP- binding proteins and induces hyperlasiaof epidermis, J,Biol Chen 1992; $267: 2600-2604$

12.Singh G,RAO DJ. Bacteriology of psoriatic plaques. Dermatogica 1978; 157(1):21-27 